\title{
Extending Timescales and Narrowing Linewidths in NMR
}

\author{
Takuya F. Segawa ${ }^{\star a}$, Aurélien Borneta, Nicola Salvi ${ }^{a}$, Pascal Miévillea, Veronika Vitzthumª, \\ Diego Carnevale ${ }^{a}$, Sami Jannina, Marc A. Caporinia, Simone Ulzega ${ }^{a}$, Paul R. Vasos ${ }^{\text {ab }}$, Martial Reya \\ and Geoffrey Bodenhausen ${ }^{\star a c}$
}

\begin{abstract}
Among the different fields of research in nuclear magnetic resonance (NMR) which are currently investigated in the Laboratory of Biomolecular Magnetic Resonance (LRMB), two subjects that are closely related to each other are presented in this article. On the one hand, we show how to populate long-lived states (LLS) that have long lifetimes $T_{\text {LLS }}$ which allow one to go beyond the usual limits imposed by the longitudinal relaxation time $T_{1}$. This makes it possible to extend NMR experiments to longer time-scales. As an application, we demonstrate the extension of the timescale of diffusion measurements by NMR spectroscopy. On the other hand, we review our work on long-lived coherences (LLC), a particular type of coherence between two spin states that oscillates with the frequency of the scalar coupling constant $J_{I S}$ and decays with a time constant $T_{\text {LLC }}$. Again, this time constant $T_{L \mathrm{C}}$ can be much longer than the transverse relaxation time $T_{2}$. By extending the coherence lifetimes, we can narrow the linewidths to an unprecedented extent. $J$-couplings and residual dipolar couplings (RDCs) in weakly-oriented phases can be measured with the highest precision.
\end{abstract}

Keywords: Diffusion · Long-lived coherences · Long-lived states · NMR · Ubiquitin

\section{Introduction}

The Laboratory of Biomolecular Magnetic Resonance (LRMB), which belongs to the Institute of Chemical Sciences and Engineering (ISIC) of EPFL, is dedicated to the development of new methods in the field of Nuclear Magnetic Resonance (NMR) both in liquid and solid samples. Our group covers more areas than can be presented in this short overview. Two important branches of our 'tree of knowledge' were recently described in CHIMIA: Simone Ulzega (SCS Mettler-Toledo Award Winner, 2009) explained our efforts to detect the highlyabundant nitrogen-14 isotope in solid samples. ${ }^{[1]}$ The challenge comes from the fact that, unlike the popular but less abundant ${ }^{15} \mathrm{~N}$ with $I=1 / 2,{ }^{14} \mathrm{~N}$ has a spin $I=1$ and a large quadrupole coupling, leading to very broad lines. ${ }^{[2,3]}$ Pascal Miéville (SCS DSM Award

${ }^{{ }^{*} \text { Correspondence: }}$ T. F. Segawa ${ }^{\mathrm{a}}$

Prof. Dr. G. Bodenhausen ${ }^{\text {ac }}$

Tel.: +41216939431

Fax: + 41216939435

E-mail: takuya.segawa@epfl.ch;

geoffrey.bodenhausen@epfl.ch

aEcole Polytechnique Fédérale de Lausanne, Institut des Sciences et Ingénierie Chimiques

Laboratoire de Résonance Magnétique Biomoléculaire, Batochime, $\mathrm{CH}-1015$ Lausanne, Switzerland

bUniversité Paris Descartes, UMR 8601 CNRS

45, rue des Saints-Pères 75006, Paris, France

'Département de Chimie, Ecole Normale Supérieure,

24 Rue Lhomond, 75231, Paris Cedex 05, France

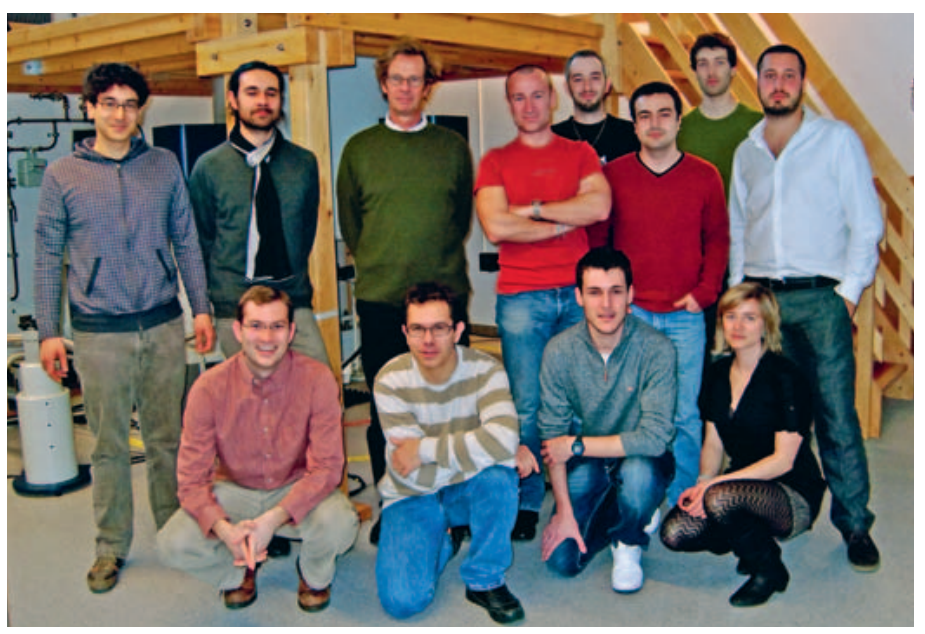

Group members of the LRMB in front of the $800 \mathrm{MHz}$ NMRspectrometer in March 2011. First row, from left: Marc Caporini, Pascal Miéville, Roberto Buratto, Veronika Vitzthum. Second row: Takuya Segawa, Diego Carnevale, Geoffrey Bodenhausen, Simone Ulzega, Aurélien Bornet, Nicola Salvi, Jonas Milani, and Sami Jannin.

Winner, 2010) et al. introduced the subject of Dynamic Nuclear Polarization (DNP), a highly promising tool for NMR since it can overcome the lack of sensitivity inherent to virtually all NMR experiments. ${ }^{[4]}$ DNP enhances NMR signals by transferring the polarization from electrons to nuclei. Currently, there are two different DNP setups at the LRMB. One comprises a polarizing magnet for DNP at a temperature of ca. $1.2 \mathrm{~K}$, followed by rapid dissolution and transfer to a liquid-state NMR spectrometer. ${ }^{[5-10]}$ This approach can reach enhancements as high as $10^{3}-10^{4}$, albeit only in one-shot experiments. The other DNP setup consists of a gyrotron that sends microwave irradiation to a solid-state NMR spectrometer equipped for magic angle spinning (MAS). The microwave irradiation saturates the electron paramagnetic resonance (EPR) transitions of radicals in a spinning solid at $100 \mathrm{~K} \cdot{ }^{[3,11,12]}$ Here, signals can be enhanced in continuous mode by a factor in the range $10-100$. With the help of both DNP setups, NMR spectra of samples can be recorded which would show nothing but noise on a normal spectrometer.

We also study spin echoes, a phenomenon that is almost as old as NMR, and try to find ways to eliminate homonuclear $J$-couplings.[13-15] This opens new paths to measure transverse relaxation times $T_{2}$ in homonuclear coupled systems, e.g. for protons, and in biomolecules that are enriched in ${ }^{13} \mathrm{C}$ or ${ }^{15} \mathrm{~N}$, where the echo decays are normally masked by $J$-modulations.

Further fields of research comprise the development of novel NMR tools to study 
chemical exchange by observing the lifetimes of heteronuclear multiple-quantum (MQ) coherences, e.g. in ${ }^{1} \mathrm{H}-{ }^{15} \mathrm{~N}$ spin pairs in proteins. ${ }^{[16-18]}$ The advantage of observing MQ relaxation rather than single-quantum (SQ) relaxation lies in the fact that it can provide information about two spins that are simultaneously affected by chemical exchange. This technique is important for the bio-NMR community since it covers the timescale from microseconds to milliseconds where many conformational motions occur that are believed to be relevant for biological processes.

There are two other fundamental parameters in NMR that have been addressed in our group in the last few years: the long memory of long-lived states (LLS), which have life times that can be much longer than the longitudinal relaxation time $T_{1},[5,7,19,20]$ and the narrow linewidths on the order of tens of millihertz associated with longlived coherences (LLC). ${ }^{[21-23]}$ This article focuses on these long-lived phenomena in NMR.

\section{Long-Lived States (LLS)}

Any NMR pulse sequence is limited in time: after an interval on the order of the longitudinal relaxation time $T_{1}$ (typically a few seconds for protons), the populations will reach the Boltzmann distribution and this will be the end of the nuclear spin memory. In 2004, Malcolm Levitt and co-workers showed a way to break this limit.[24,25] By applying a simple radio frequency $(r f)$ pulse sequence, they increased the spin memory several times beyond $T_{1}$. Since relaxation is usually determined by molecular structure and motion, and is normally impervious to $r f$ pulses, this discovery is very exciting.

Consider a system of two spins-1/2 named $I$ and $S$ with different chemical shifts $v_{I}$ and $v_{S}$ and a scalar coupling of $J_{I S}$. We assume that they are reasonably isolated from other nuclei with significant magnetic moments. The normal NMR spectrum of such a weakly coupled system shows two doublets split by $J_{I S}$ and centered at the two chemical shifts $v_{I}$ and $v_{S}$. ${ }^{[26]}$ The corresponding energy-level diagram is shown on the left side of Fig. 1, with four possible product states $\alpha \alpha, \alpha \beta, \beta \alpha$ and $\beta \beta$. If the difference between the two chemical shifts where nil (i.e. if we had two magnetically equivalent spins) the energy-level diagram would change to the one on the right side in Fig. 1, and the spectrum would show a singlet. While the highest and lowest levels $\alpha \alpha$ and $\beta \beta$ stay unchanged, the middle ones are made up of a sum and a difference of $\alpha \beta$ and $\beta \alpha$ (with the normalization $N=2^{-1 / 2}$ ). The four states can be classified in a triplet and a singlet: the

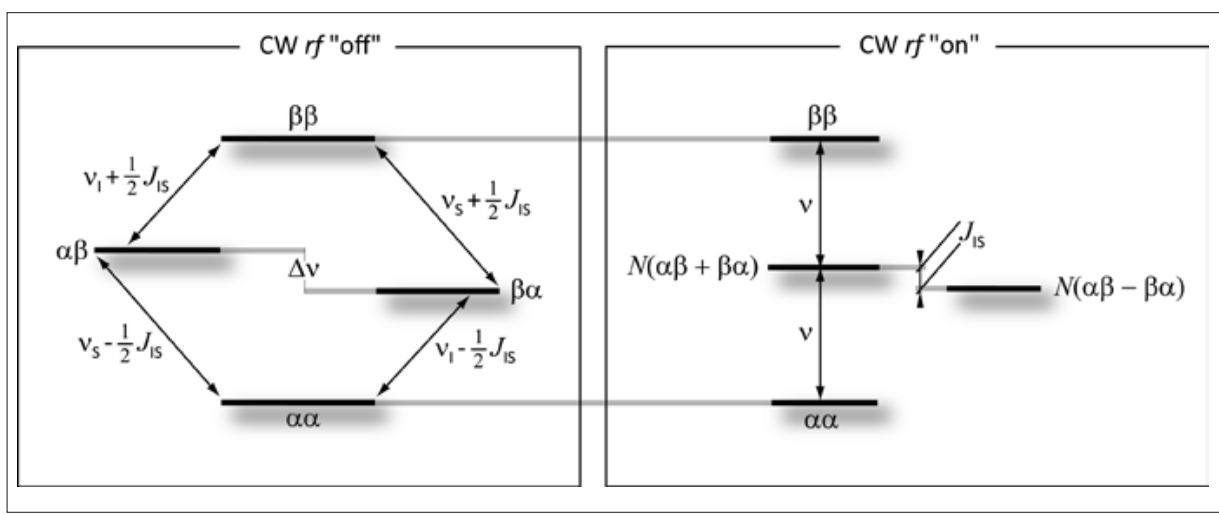

Fig. 1. Graphical representations of the two energy-level diagrams (not to scale), used for the creation of long-lived states (LLS) and long-lived coherences (LLC). (Left) The energy-level diagram of two spins $/$ and $S$ with two different chemical shifts $v_{l}$ and $v_{S}$. The four possible products $\alpha \beta$, $\alpha \beta, \beta \alpha$ and $\beta \beta$ constitute the states for this system. The arrows show the single-quantum (SQ) transitions that give rise to peaks in an ordinary NMR spectrum. (Right) The energy-level diagram of two magnetically equivalent spins (i.e. both spins have identical chemical shifts $v$ ). A simple NMR spectrum shows only one peak at the frequency $v$. The energy levels $\alpha \alpha$ and $\beta \beta$ are identical in both systems. The middle ones are normalized sums and differences of $\alpha \beta$ and $\beta \alpha$, with an energy difference $J_{I S}$. The three exchange-symmetric states $\alpha \alpha, \beta \beta$ and $N(\alpha \beta+\beta \alpha)$ constitute a triplet, while the exchange-antisymmetric state $N(\alpha \beta-\beta \alpha)$ is a singlet (with the normalization $N=2^{-1 / 2}$ ). By applying a continuous-wave radiofrequency $(r f)$ irradiation, chemical shifts can be suppressed and the system on the left-hand side can be converted into the system on the right-hand side with $v=\left(v_{1}+v_{s}\right) / 2$. The energy difference between $N(\alpha \beta+\beta \alpha)$ and $N(\alpha \beta-\beta \alpha)$ of $J_{\text {IS }}$ determines the frequency at which the LLC oscillates.

three states $\alpha \alpha, \beta \beta$ and $N(\alpha \beta+\beta \alpha)$, which are unaffected by the permutation of the two spins, constitute the triplet manifold, while $N(\alpha \beta-\beta \alpha)$, which switches sign under permutation of the two spins, constitutes the singlet state. If there is only one chemical shift, nothing but relaxation can connect the singlet and the triplet states. However the relaxation mechanism itself should be exchange-antisymmetric. This is not the case for the dominant homonuclear dipole-dipole coupling of the two spins. Therefore the population of a singlet state can persist over very long intervals, much longer than the usual longitudinal relaxation time $T_{1}$. The singlet state is not magnetic since its total nuclear spin vanishes and therefore, it cannot be directly manipulated. That is why we need an inequivalent $\mathrm{AX}$-system both to populate the singlet state at the beginning of the experiment and to detect it at the end. The job of the $r f$ pulse sequence is first to populate the singlet state, then to switch to magnetic equivalence by suppressing the difference in chemical shifts (i.e. going from left to right in Fig. 1), and, finally, to convert the population of the singlet state into observable magnetization. The second step is fulfilled by on-resonance continuous-wave $r f$ irradiation, in the manner of decoupling. Further developments of pulse sequences led to lifetimes $T_{\text {LLS }}$ that were $c a .36$ times longer than $T_{1}$ in the case of a proton pair in a partially deuterated sugar.[27]

This has opened up new timescales for NMR experiments. As an example, we will present measurements of translational diffusion with the help of NMR. ${ }^{[28,29]}$ By using pulsed field gradients (PFGs), the homogeneity of the magnetic field (which is normally optimized by tedious shimming!) is destroyed, but in a controlled way. Even though the spins are dephased over the whole sample, if the gradient is applied along the vertical z-axis, they are in-phase within a slice at a given height. If we apply a second PFG with an opposite sign, the coherence can be restored over the whole sample. This is only true if the molecules and their spins do not move between the two PFGs. If we vary the delay $\Delta$ between the PFGs (or better, the strength of the gradient $G$ ), the effect of diffusion can be measured. This delay $\Delta$ was limited till now by $T_{1}$. By using long-lived states in the diffusion experiment, the delay $\Delta$ can be extended since $T_{\mathrm{LLS}} \gg T_{1}$. This makes macromolecules $(>50 \mathrm{kDa})$ with small diffusion coefficients $\left(D<10^{-10} \mathrm{~m}^{2} \mathrm{~s}^{-1}\right)$ accessible. ${ }^{[30,31]}$ Ideally, the macromolecule should contain a sub-system of two spins with a magnetization that can be converted to a singlet state. ${ }^{[20]}$ Glycine residues in peptides and proteins are suitable systems. The aliphatic $\mathrm{CH}^{\alpha}{ }_{2}$ protons in monomeric glycine are magnetically equivalent, but become diastereotopic when bound to chiral amino acids. In the protein ubiquitin (molecular mass $8.5 \mathrm{kDa}$ ), without any isotope labeling, it is possible to excite LLS with $T_{\mathrm{LLS}} / T_{1}>6$ in the two highly mobile Gly-75 and Gly-76 at the C-terminus, which are fairly remote from the bulk of the protein. Fig. 2 shows the comparison of two diffusion experiments, one using $T_{\text {LLS }}$ 


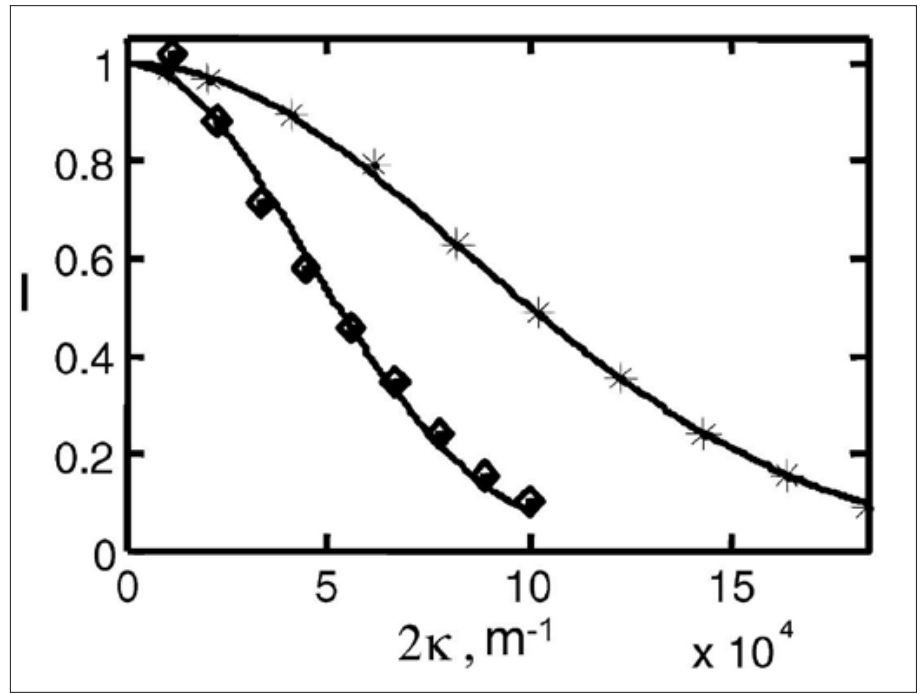

Fig. 2. Fits of diffusion coefficients of ubiquitin dissolved in $\mathrm{D}_{2} \mathrm{O}$ at $T=$ $298 \mathrm{~K}$ and $\mathrm{B}_{0}=11.7 \mathrm{~T}$ ( ${ }^{1} \mathrm{H}$ frequency of $500 \mathrm{MHz}$ ) using long-lived states (LLS) (diamonds) and conventional longitudinal magnetization (stars). The diffusion intervals were $\Delta_{u S}=2 \mathrm{~s}$ and $\Delta_{1}=0.55 \mathrm{~s}$, respectively. The gradient strength $G$ must be much higher if one uses longitudinal magnetization than if one employs LLS. Both experiments lead to similar diffusion coefficients $D=(1.264 \pm 0.002) \times 10^{-10} \mathrm{~m}^{2} \mathrm{~s}^{-1}$. (Reprinted with permission from ref. [20]. Copyright 2009 by the American Chemical Society.)

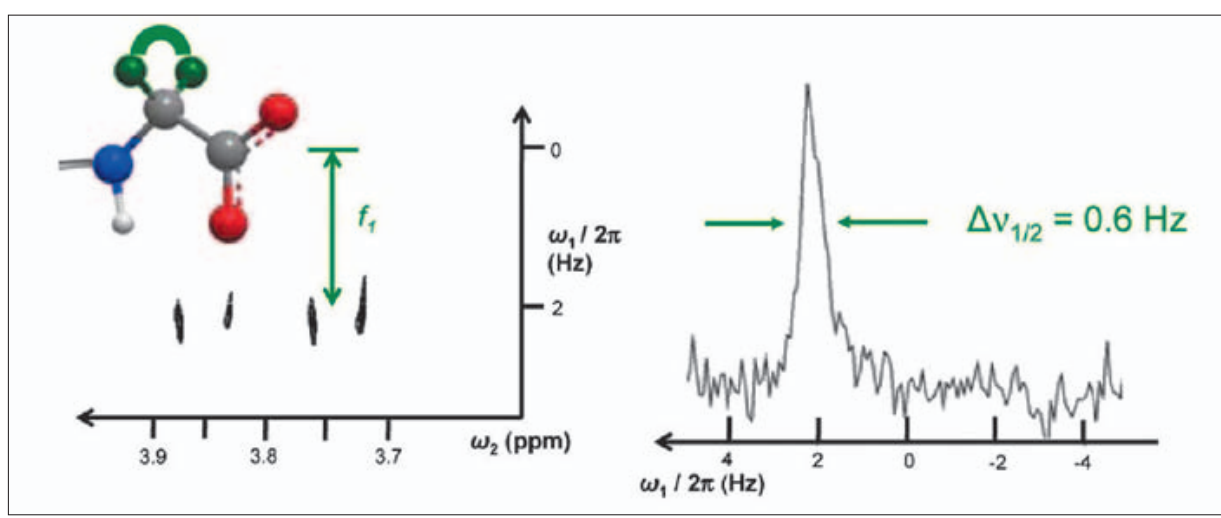

Fig. 3. Observation of a long-lived coherence (LLC) in a two-dimensional (2D) proton spectrum of ubiquitin with very narrow linewidths in the (indirect) $\omega_{1}$ dimension at $T=300 \mathrm{~K}$ and $\mathrm{B}_{0}=9.4 \mathrm{~T}$ ( ${ }^{1} \mathrm{H}$ frequency of $400 \mathrm{MHz}$ ). (Left) 2D spectrum of the LLC comprising two inequivalent protons $\mathrm{H}^{\alpha}$ (green spheres) of the terminal amino acid Gly-76 in the protein ubiquitin. The spectral window was reduced to $10 \mathrm{~Hz}$ in the $\omega_{1}$ dimension (only $3 \mathrm{~Hz}$ shown), so that the folded peaks appear at $2.17 \mathrm{~Hz}$, corresponding to $J_{I S}=-17.17 \mathrm{~Hz}$. (Right) Cross section extracted from the spectrum on the left-hand side at $\omega_{2}=3.84 \mathrm{ppm}$; the linewidth in the $\omega_{1}$ dimension is 0.6 . Hz. (Reprinted with permission from ref. [21]. Copyright 2010 by the American Physical Society.)

(diamonds) and the other $T_{1}$ (stars). While the $T_{\text {LLS }}$ experiment can tolerate a longer diffusion interval of $\Delta_{\mathrm{LLS}}=2 \mathrm{~s}$, the $T_{1}$ experiment is restricted to $\Delta_{1}=0.55 \mathrm{~s}$, since spin memory is severely attenuated beyond this horizon. Here, the diffusion interval $\Delta$ is kept constant, but the gradient strength is varied, so as to vary the product $\kappa=\delta \gamma p s G$ where $\gamma$ is the proton gyromagnetic ratio, $p$ $=1$ is the coherence order, $\delta$ is the gradient duration, and $s=2 / \pi$ is a shape factor that describes the shape of the gradients, and $G$ the gradient strength. The $T_{1}$ experiment needs stronger gradients (since $\Delta$ must be

While LLS deal with populations, the the lifetimes of long-lived coherences. The decay of ordinary 'single quantum' relaxation times $T_{2} \leq T_{1}$. Usually, the apparent linewidth $R_{2}^{*}$ is broader than $R_{2}=$ $1 / T_{2}$ because of inhomogeneous broadening. The contribution stemming from the inhomogeneity of the magnetic field can be removed by forming echoes, the simplest way to measure $T_{2}$. Reducing the underlying homogeneous broadening is more challenging. Generally speaking, the larger the molecule, the slower the rotational diffusion, the faster the transverse relaxation, and the broader the spectral lines.

Going back to the right side of Fig. 1, Levitt and co-workers ${ }^{[32]}$ and Sarkar et al. ${ }^{[21]}$ recently found ways to create a coherent superposition of the singlet and the central triplet states: a long-lived coherence (LLC). The evolution of an LLC can be monitored in the indirect dimension of a two-dimensional (2D) experiment, or 'on the fly' in a 1D experiment. In contrast to the case of LLS, LLCs do not simply decay according to a mono-exponential function, but oscillate with the frequency of the scalar coupling $J_{I S}$. This is due to the energy difference between the two involved states, which is exactly $J_{I S}$. The relaxation time constant $T_{\text {LLC }}$ of the LLC is often much longer than the usual transverse relaxation time $T_{2}$. By applying a Fourier transform to the oscillatory decay, we obtain two peaks at frequencies $\pm J$ which are very narrow, thanks to the long lifetime. An example of such a spectrum is shown in Fig. 3. Using the same system as presented above, the two $\mathrm{H}^{\alpha}$ protons in the terminal Gly-76 of ubiquitin (green in Fig. 3), a 2D LLC experiment is shown on the left side of Fig. 3 . In the direct (horizontal) $\omega_{2}$ dimension we see a normal spectrum showing two doublets at two chemical shifts; in the indirect (vertical) $\omega$ dimension, the frequency corresponds to the value of the $J$-coupling. The peaks are folded in the indirect dimension and appear at $2.17 \mathrm{~Hz}$, which corresponds in reality to $J_{I S}=-17.17 \mathrm{~Hz}$. The right-hand side of Fig. 3 shows a cross section extracted from the spectrum at $\omega_{2}$ $=3.84 \mathrm{ppm}$. The linewidth in the $\omega_{1}$ dimension is $0.6 \mathrm{~Hz}$. The current record for the smallest linewidth of a organic molecule in our lab is as narrow as $14 \mathrm{mHz}$.

This experiment is not only a tool to measure $J$-couplings with unprecedented precision. In the case of two overlapping chemical shifts, the second $J$-dimension may help to discriminate them. In weakly oriented phases, one can measure residual dipolar couplings (RDCs). ${ }^{23]}$ These splittings contain information about distances and order parameters and are widely used in the determination of three-dimensional structures and dynamics of biomolecules. second part of this article is dedicated to coherences is characterized by transverse

\section{Acknowledgments}

This article is based in part on research performed by two former $\mathrm{PhD}$ students of the 
LRMB: Dr. Riddhiman Sarkar and Dr. Puneet Ahuja. This work was supported by the Swiss National Science Foundation, the Commission pour la Technologie et l'Innovation (CTI), the Ecole Polytechnique Fédérale de Lausanne (EPFL), and the French CNRS.

Received: March 28, 2011

[1] S. Ulzega, Chimia 2010, 64, 157.

[2] S. Cavadini, V. Vitzthum, S. Ulzega, A. Abraham, G. Bodenhausen, J. Magn. Reson. 2010, 202, 57

[3] V. Vitzthum, M. A. Caporini, G. Bodenhausen, J. Magn. Reson. 2010, 205, 177

[4] P. Miéville, S. Jannin, L. Helm, G. Bodenhausen, Chimia 2011, 65, 260.

[5] P. R. Vasos, A. Comment, R. Sarkar, P. Ahuja, S Jannin, J. P. Ansermet, J. A. Konter, P. Hautle, B. van den Brandt, G. Bodenhausen, Proc. Natl. Acad. Sci. USA 2009, 106, 18469.

[6] R. Sarkar, A. Comment, P. R. Vasos, S. Jannin, R. Gruetter, G. Bodenhausen, H. Hall, D. Kirik, V. P. Denisov, J. Am. Chem. Soc. 2009, 131, 16014.

[7] P. Ahuja, R. Sarkar, S. Jannin, P. R. Vasos, G. Bodenhausen, Chem. Commun. 2010, 46, 8192.

[8] A. Comment, S. Jannin, J. N. Hyacinthe, P. Mieville, R. Sarkar, P. Ahuja, P. R. Vasos, X. Montet, F. Lazeyras, J. P. Vallee, P. Hautle, J. A. Konter, B. van den Brandt, J. P. Ansermet, R. Gruetter, G. Bodenhausen, Phys. Rev. Lett. 2010, 105, 018104 .

[9] P. Mieville, P. Ahuja, R. Sarkar, S. Jannin, P. R. Vasos, S. Gerber-Lemaire, M. Mishkovsky, A. Comment, R. Gruetter, O. Ouari, P. Tordo, G. Bodenhausen, Angew. Chem. Int. Ed. 2010, 49 , 6182.

[10] P. Mieville, S. Jannin, L. Helm, G. Bodenhausen, J. Am. Chem. Soc. 2010, 132, 5006.

[11] A. Lesage, M. Lelli, D. Gajan, M. A. Caporini, V. Vitzthum, P. Mieville, J. Alauzun, A. Roussey, C. Thieuleux, A. Mehdi, G. Bodenhausen, C. Coperet, L. Emsley, J. Am. Chem. Soc. 2010, 132, 15459 .

12] M. Lelli, D. Gajan, A. Lesage, M. A. Caporini, V. Vitzthum, P. Miéville, F. Héroguel, F. Rascón,
A. Roussey, C. Thieuleux, M. Boualleg, L. Veyre, G. Bodenhausen, C. Copéret, L. Emsley, J. Am. Chem. Soc. 2011, 133, 2104.

[13] B. Baishya, T. F. Segawa, G. Bodenhausen, J. Am. Chem. Soc. 2009, 131, 17538.

[14] T. F. Segawa, N. Aeby, G. Bodenhausen, Phys. Chem. Chem. Phys. 2010, 12, 9772.

[15] T. F. Segawa, B. Baishya, G. Bodenhausen, ChemPhysChem 2010, 11, 3343.

[16] M. Verde, S. Ulzega, F. Ferrage, G. Bodenhausen, J. Chem. Phys. 2009, 130, 074506.

[17] S. Ulzega, M. Verde, F. Ferrage, G. Bodenhausen, J. Chem. Phys. 2009, 131, 224503.

[18] S. Ulzega, N. Salvi, T. F. Segawa, F. Ferrage, G. Bodenhausen, ChemPhysChem 2011, 12, 333.

[19] P. Ahuja, R. Sarkar, P. R. Vasos, G. Bodenhausen, ChemPhysChem 2009, 10, 2217.

[20] P.Ahuja, R. Sarkar, P. R. Vasos, G. Bodenhausen, J. Am. Chem. Soc. 2009, 131, 7498 .

[21] R. Sarkar, P. Ahuja, P. R. Vasos, G. Bodenhausen, Phys. Rev. Lett. 2010, 104, 053001.

[22] A. Bornet, R. Sarkar, G. Bodenhausen, J. Magn. Reson. 2010, 206, 154.

[23] R. Sarkar, P. Ahuja, P. R. Vasos, A. Bornet, O. Wagnières, G. Bodenhausen, Prog. Nucl. Magn. Reson. Spectrosc. 2011, 59, 83.

[24] M. Carravetta, O. G. Johannessen, M. H. Levitt, Phys. Rev. Lett. 2004, 92, 153003.

[25] M. Carravetta, M. H. Levitt, J. Am. Chem. Soc. 2004, 126, 6228

[26] R. Freeman, 'Spin choreography: Basic Steps in High Resolution NMR'; OUP, USA, 1998.

[27] R. Sarkar, P. R. Vasos, G. Bodenhausen, J. Am. Chem. Soc. 2007, 129, 328.

[28] E. O. Stejskal, J. E. Tanner, J. Chem. Phys. 1965, 42, 288.

[29] C. S. Johnson, Prog. Nucl. Magn. Reson. Spectrosc. 1999, 34, 203

[30] S. Cavadini, J. Dittmer, S. Antonijevic, G. Bodenhausen, J. Am. Chem. Soc. 2005, 127, 15744.

[31] S. Cavadini, P. R. Vasos, Concepts in Magnetic Resonance Part A 2008, 32A, 68.

[32] G. Pileio, M. Carravetta, M. H. Levitt, Phys. Rev. Lett. 2009, 103, 083002 